\title{
The Feature of Animation Role Mold in Animation Film from Monkey King: Hero Is Back \\ Chuang Chen
}

School of Art, Zhengzhou University of Industry Technology, Zhengzhou, China 474570630@qq.com

Keywords: Animation; Role mold; Presupposition; Virtualization

\begin{abstract}
As the core of animation film,animated characters have different feathers, To analyze and understand animation role mold,the most important is grasp the differences between animation film and real movie.The feature of animated characters is presupposition and virtualization. As an important branch of the film and the requirement of art and technological development, animation role mold have greatly enriched film character building system. In this paper, analyze animation role mold through the differences between animation film and real movie based on the animation film Monkey King: Hero Is Back.
\end{abstract}

\section{从《大圣归来》看动画电影角色塑造之特性}

陈闯

郑州工业应用技术学院艺术学院，中国河南省郑州市 451100

474570630@qq.com

摘要: 动画角色作为动画电影的核心, 具有独特的特点, 要分析和认识动画角色塑造的规律, 最重要的是掌握动画电影角色和实拍电影角色塑造的区别。动画电影角色的特性主要表现在 预设性和虚拟性。作为电影的一个重要分支, 由于艺术的要求和技术的发展, 动画角色塑造 一定程度上大大丰富了电影角色塑造体系。在本文中, 通过对《大圣归来》的分析, 对比动 画角色塑造和实拍电影角色塑造的区别，阐述动画电影角色塑造的特性。

关键词：动画；角色塑造；预设性；虚拟性

引言

动画作为一门艺术形式, 其构成元素具有明显的符号化特征, 通过元素的有机组合, 构成了 信息的传递系统 [1]。电影的符号编码系统，就像文学一样通过字、词、句的有机组合，向人 们传递电影语言, 动画角色作为动画电影的 “演员” , 在动画中居于核心地位, 将动画角色 通过艺术加工和构思达到叙述故事, 传递信息的目的。所以说研究动画电影角色塑造的特性, 要从动画角色本身入手，区别动画电影角色塑造和实拍电影角色塑造，动画角色塑造的特性 主要表现在预设性和虚拟性。

动画角色塑造，比实拍电影角色塑造更能适合人们的心理，伴随着技术和艺术的发展，动画 电影角色塑造出现多样化和精细化, 本文以《大圣归来》为分析基础, 从预设性和虚拟性两 方面阐述动画角色塑造特性。

\section{1. 动画角色塑造的预设性}

动画电影不同于实拍电影就在于其预设性，动画导演拍摄的依据是动画分镜头剧本，在动画 分镜头剧本中已经详细的说明了摄像机运动、人物运动、人物对白等一系列制作中需要的内 容, 动画角色的塑造正是动画分镜头剧本制作的核心环节, 没有动画角色, 动画分镜头剧本 
就无法进行设计, 而动画角色塑造和实拍电影角色塑造不同, 动画角色塑造具有预设性 [2]。 动画角色塑造的预设性体现在以下几个方面: 动画角色生活背景预设、动画角色的造型预设、 动画角色性格预设等。动画角色的塑造是动画艺术家根据动画剧情需要, 通过大量的素材收 集提炼, 再经过创意等艺术加工阶段的产物, 动画角色在一定程度来讲是动画艺术家的影子, 动画艺术家会根据需要将自己的理解加入到动画角色塑造中去, 动画角色按照动画的发展和 动画艺术家的要求去进行 “表演” , 实拍电影则根据剧本选择演员进行表演拍摄, 在人物的 塑造上除了按照剧本设置之外, 演员自身的素质将会对角色塑造起到较大作用, 动画角色塑 造则不然, 动画角色可以忠实的按照动画导演的要求进行 “表演” [3]。

1.1. 动画角色生活背景的预设性

动画角色是动画艺术家创造出来的产物, 动画艺术家要根据动画故事的发展对动画角色的生 活背景进行预设, 动画角色的生活背景对其造型、性格、故事的发展走向都有较大影响。[4] 通过动画角色生活背景的预设, 可以使动画角色设计人员快速掌握动画角色的基本情况, 按 照动画角色的设计规律进行设计 [4]。

《大圣归来》中, 孙悟空的生活背景就设计为一个失去法力的孙悟空, 孙悟空甚至具备人和 猴子的双重性格, 而唐僧也不再是我们在实拍电视剧中看到中年人的形象, 而是一个孩童形 象, 所以动画角色预设给动画设计师提供了设计的基本要求。

\section{2. 动画角色造型预设性}

动画角色造型是以绘画的手法来表现动画角色主体造型和空间造型, 动画角色造型具有高度 的假定性, 动画角色的造型可以用夸张、变形、拟人等手法进行设计, 动画角色造型可以表 达作者的理想和想象, 所以动画角色造型具有实拍电影无法比拟的优势 [5]。但动画角色造型 设会严格依据动画剧本的要求进行设计, 同时动画设计师根据剧本进行再创造, 将自己的理 想和想象注入到动画角色造型中, 使角色成为有血有肉的人物, 动画角色造型这些特点也决 定了其预设性。

《大圣归来》的故事不再是我们看到的师徒四人取经的故事, 而是小和尚和小猴子五行山下 寂寞沉潜五百年的孙悟空被儿时的唐僧一俗名江流儿的小和尚误打误撞地解除了封印, 在相 互陪伴的冒险之旅中找回初心, 完成自我救赎的故事。孙悟空的形象也为观众提供了新的审 美方向, 孙悟空失去了红心脸, 将脸部轮廓设计的瘦削刚硬, 同时加入了皱纹以加强人物的 沧桑感, 整体形象则混搭了长臂猿的形象, 最终变成了一名 “长脸失意大叔”。而片中的土 地公公也设计成土拔鼠，反面人物妖怪设计成为书生道士。

1.3. 动画角色性格预设性

动画角色性格是指动画角色在动画片中展现出的独特气质和品格, 动画角色的性格是动画设 计师根据动画剧本和动画故事发展需要赋予动画角色的, 所以在动画制作的前期阶段就会先 将动画角色性格进行设计, 这也就决定了动画角色性格的预设性 [6]。

《大圣归来》中的孙悟空不再是具有无穷法力的齐天大圣, 而是被封印下的猴子, 显示出的 是无奈和落寞的性格, 同时带有风趣和幽默, 具有了人的特性, 将英雄赋予了更人性化的特 点; 唐僧是幼年的小孩子, 对任何事情都十分好奇, 对英雄充满了崇拜, 于是就设计成为一 个话唠的形象，不停的对孙悟空进行追问。

\section{2. 动画角色塑造的虚拟性}

动画是具有高度假定性的艺术, 动画可以运用夸张、拟人、变形等手法, 借助于幻想、想象 和象征, 反应人们的思想, 动画更容易直观表达和抒发人们的感情, 动画具有虚拟性的特点, 包括虚拟的剧情、虚拟的角色、虚拟的时空、虚拟的声音等, 动画角色作为动画电影的 “演 员” ，通过动画角色的 “表演” 推动故事发展的任务, 动画角色塑造的虚拟性表现在动作的 虚拟和 “表演” 的虚拟 $[7]$ 。

2.1. 动作的虚拟性

在动画创作中, 动画角色是根本不存在的, 是动画艺术家创造的产物, 无论是二维动画角色 
还是三维动画角色都是不存在的, 动画艺术家根据动画剧本要求设计出动画角色, 加入了设 计师的理想和想象, 很大程度来讲动画角色是动画设计师的精神寄托。动画角色在动画故事 的发展中, 承担着和实拍电影演员一样的任务: 通过动作和语言推动故事的发展。实拍电影 的演员通过自身的动作和语言进行实际表演, 而动画电影角色的动作则是通过动画设计师设 计出来的, 是动画设计师对动作理解后的创造产物, 所以说动画角色的动作具有虚拟性 [8]。

《大圣归来》中, 孙悟空的原型是一只猴子, 而在剧中他直立行走, 潇酒自然, 面部表情丰 富, 情感流露真实, 例如在孙悟空发现自己法力全失的时候的那种落寞和无奈, 通过弯曲的 身体表现的淋漓尽致; 剧中将一只土拔鼠通过拟人的手法设计成土地公公, 通过蚌萌的动作 给观众留下了深刻的印象; 唐僧设计成为一个小孩子, 蹦蹦跳跳的动作, 天真烂漫的表情, 获得了观众的喜爱。很大程度来说这些动作和表情是动画设计师智慧的结晶。

2.2. 表演的虚拟性

动画角色的表演是决定动画成功与否的关键，如果忽视动画角色表演，就算拥有高超的技术 也很难给观众留下深刻的印象。表演主要指表演者利用技艺或专长来传达具体的事件或非具 体的意象, 以达到艺术或是娱乐的目的。表演的形式有很多, 例如戏剧表演、舞蹈表演、影 视表演，当然还有动画表演，各种表演都塑造角色，但展现出的艺术特征却不同 [9]。 动画表演是是指以故事剧本为基础, 以动画角色为核心, 通过动画角色运用夸张、变形等手 法演绎故事，以更好地塑造角色的情绪和个性。动画角色表演不同于其它形式的艺术表演， 它是一种虚拟性的表演, 同真人表演不同。动画表演的主体是动画角色, 而动画角色是由动 画导演和原画师构思出来的虚拟形象。动画角色本身没有生命, 只有通过动画师的艺术加工, 才能让表演为动画角色注入了灵魂, 动画角色按照动画剧本和动画设计师的要求进行忠实的 表演, 而实拍电影的角色除了按照剧本表演之外, 演员自身的素质也对角色的塑造起到很大 的作用。

表演分为 “体验派” 和 “表现派” ，体验派的表演基础对角色的理解，动画设计师将自己想 象为角色本身, 并进入角色所在的世界, 亲身体现角色的感受和内心世界, 由内而外的做出 动作和反应，进而进行设计；动画角色表演同时也具备表现派的特征，把自己体验角色时所 表现出的肢体语言记录下来然后再进行设计。所不同的是动画角色表演动作和表情更夸张， 所以动画表演更能符合人们的心理期望，更淋漓尽致 [10]。

《大圣归来》中, 为了设计孙悟空的动作, 动画设计师专门观看了猴子的生活, 通过观察提 出猴子的动作特征, 然后经过动画设计师自身的理解进行提炼, 最终把动作注入到孙悟空这 个角色中, 使孙悟空具备了猴子和人的双重性格, 使大圣具备了亲民的特质。无论是孙悟空 还是唐僧、猪八戒这些角色还是其他的动画人物, 他们在现实世界中并不存在, 他们的动作 也是动画设计师经过创造的出来的, 具有虚拟性, 而正是这种虚拟性的特性, 吸引了观众的 兴趣, 获得了观众的认可。

\section{3. 结语}

无论是动画电影还是实拍电影, 其本质都是叙事, 只是在角色的塑造上采取的方式不同, 动 画角色塑造渗透着动画设计师的智慧, 正是动画角色具备的预设性和虚拟性, 给了动画设计 师想象和创造的空间, 我们才有了这些具备独特气质的动画角色, 通过他们的表演, 得到更 符合观众心理预期的效果, 动画角色其实就是动画电影的 “演员”。动画电影作为电影的重 要分支, 共同承担着大众传播的责任, 对于动画发展进行适当的总结分析, 有助于动画片的 发展, 对动画角色塑造的特性研究, 有助于我们更好的理解动画角色, 有利于我们的动画创 作。

\section{参考文献}

[1] 高琳, 从飞屋环游记看动画电影视听语言之特性 $[\mathrm{J}]$. 电影文学, (16)2010, 30-31. 
[2] 范俊. 对动画片中分镜头台本的分析 $[J]$. 戏剧之家, (8)2014,218-219.

[3] 米高峰, 周杰. 试论影视动画中的动画表演创作 $[J]$. 电影文学，(1)2011，63-64.

[4] 胡国锋, 动画角色造型和受众特点的关系分析 [J]. 电影文学，(5)2014,60-61.

[5] Chen Peng. Effects of characteristic motions in animation film [J].Movie iterature.2015, No.3, p. 83-85.

[6] Teng Haoquan. Discussion on the influence of role modeling design on character shaping [J]. Film Review.2011, No.1, p. 15-16.

[7] Wand Jing. Analysis of the influence of the "virtual" in the cartoon on the aesthetic education [J]. Art \& Design ,.2011, No.5, p. 120-121

[8] Wu Jian. Discussion in action design of animation role performance [J]. Art Criticism. 2013, No.3, p. 13-116.

[9] 康康. 浅谈动画表演 [J]. 美术教育研究，(5)2015,83-84.

[10]Lu Qing. The" experience" performance of the cartoon character [J].Art Review.2015, No. 10, p. 127-129.

\section{References}

[1] Gao Lin. The feature of animation audio-visual language in Animation Film from Up [J]. Movie literature.2010, No. 16, p. 30-31.

[2] Fan Jun. Analysis on the storyboard of animation [J].Home of drama.2014, No. 8, p. 218-219.

[3] Mi Gaofeng, Zhou Jie. Creation of animation performance in film and television animation [J].Movie literature.2011, No.1, p. 63-64.

[4] $\mathrm{Hu}$ Guofeng. Analysis of the relationship between the character of the cartoon and the audience [J].Movie literature.2014, No.5, p. 60-61.

[5] Chen Peng. Effects of characteristic motions in animation film [J].Movie iterature.2015, No.3, p. 83-85.

[6] TENG Haoquan. Discussion on the influence of role modeling design on character shaping [J]. Film Review.2011, No.1, p. 15-16.

[7] Wang Jing. Analysis of the influence of the "virtual" in the cartoon on the aesthetic education [J]. Art \& Design ,.2011,No.5,p. 120-121

[8] Wu Jian. Discussion in action design of animation role performance [J]. Art Criticism, 2013, No.3, p. 13-116.

[9] Kang Kang. Talking about the animation performance [J].Art Education Research, 2015, No.5, p. 83-84.

[10]Lu Qing. The" experience" performance of the cartoon character [J]. Art Review. 2015, No.10, p. 127-129. 